\title{
STATUS OF THE PRODUCTION ELECTROPOLISHING SYSTEM AT JLAB*
}

\author{
J. Mammosser\#, J. Delayen, J. Gordon, L. Phillips, A. M. Valente, T. Wang, A.T. Wu, Jefferson Lab, \\ Newport News, VA 23606, USA
}

J. Saunders, SNS, Oak Ridge, TN 37830, USA

\begin{abstract}
Jefferson Lab has installed, and is in the process of commissioning, a production electropolish system, sized for $805 \mathrm{MHz}$ SNS cavities. This paper describes the basic system design, plans for studying the effectiveness of polishing SNS high- $\beta$ cavities and early results from cavity tests.
\end{abstract}

\section{INTRODUCTION}

Historically, producing high field and Q-values in superconducting RF cavities of niobium has required the removal of a substantial layer of the inner cavity surface, typically of the order of 100 microns in depth. Two methods have commonly been used: electropolishing (EP) or immersion in a buffered chemical polish (BCP)[1]. Both methods have occasionally produced cavities exhibiting exceptionally good performance. However, some studies have demonstrated, by direct comparison under controlled conditions, substantial advantages of EP over BCP $[2,3,4]$, both in terms of Q-value and accelerating gradient. To take advantage of high performance levels obtainable with this technique, a system was installed at Jefferson Lab for the preparation of SNS cavity surfaces. A highly valuable feature of this system (Figure 1) is that it is fully automated, providing a high level of process repeatability. An additional advantage of process automation is the ability to study the process under controlled conditions, providing opportunity for rapid process optimisation and improvement. Although the reasons for the superior performance of EP over BCP are not fully understood, electropolishing produces smoother surfaces, which could conceivably increase both Q-values and maximum gradients by mechanisms suggested by Knobloch et. al. [5]. Typical values for surface roughness in one study are given as 1.5 microns for $\mathrm{BCP}$ and 0.2 microns for $\mathrm{EP}$ samples from the same material [6]. These are small sample studies, which might not reflect varying conditions over a cavity surface, such as differing current densities or electrolyte velocity between iris and equator especially for the deep cells of SNS cavities.

We have instituted a program with the following goals: commission the EP system; establish the adequacy of the EP system for SNS cavity surface preparation; and process SNS cavities for production. The commissioning phase has been successfully completed.

*Work supported by United States Department of Energy under contract DE-AC05-84ER40150

\#mammosse@jlab.org.

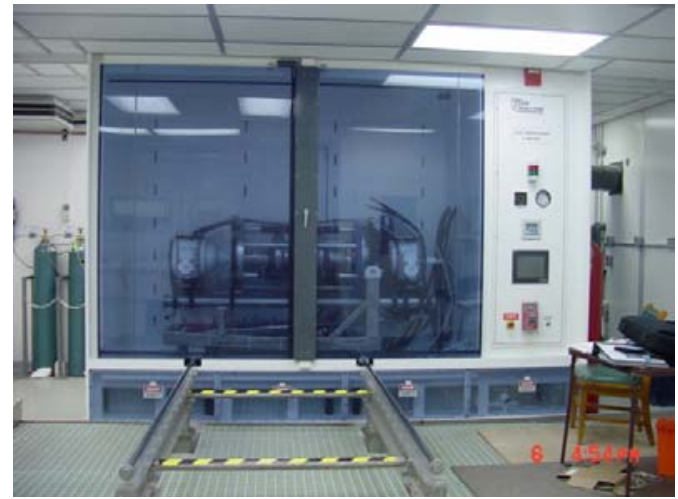

Figure 1. EP System at Jefferson Lab.

The first prototype cavity result shown in figure 3, exceeds SNS requirements for high beta cavities and establishes the effectiveness of the system.

Production processing will start when the cavities begin to arrive in June. An additional goal of the program is to understand many of the issues referred to above, which are relevant to cavity performance and specifically to SNS cavities. A prototype cavity with replaceable niobium buttons (HB-button), has been fabricated and tested and will be used to measure local current densities, roughness and chemistry of the niobium surface, etc., at different cavity locations. Such understanding is valuable in achieving the highest levels of performance possible.

\section{SYSTEM DESIGN}

The electropolishing system consists of a self-contained processing cabinet, a remotely located chiller and direct current power supply variable up to 600 amperes and 50 volts. The process cabinet is installed in one of the production process rooms, next to the cavity production cleanroom complex in the Testlab, Building 58, at Jefferson Lab. The EP cabinet was procured from industry [7] and consists of three separate compartments: plumbing, electronic and main process chamber. The plumbing compartment houses the pumping systems, valves, acid sump and instrumentation for process control. The electronic compartment contains the main process programmable logic controller and all electronic controls and wiring. The main process chamber (Figure 2) has a frame, designed for inserting and holding the cavity tilt/rotation tooling. This frame transfers the weight of the tooling to the floor and has two insertion rails that penetrate the front of the cabinet for insertion of the tooling into the process chamber. The insertion rails extend eight feet $(2.44 \mathrm{~m})$ in front of the main process chamber doors, allowing for assembly and disassembly of 
cavities into and out of the tilt/rotation tooling. The cabinet has a sub-frame that is constructed of stainless steel tubing wrapped with polypropylene plastic. All cabinet walls and surfaces are fabricated from fireretardant polypropylene sheet. The rotary sleeves that allow for horizontal rotation of the cavity during processing and serve as a primary seal. The rotary sleeves, cavity holding frame and the cathode were purchased from industry [8]. All other tooling was designed and built at Jefferson Lab.

\section{System Features}

The Jefferson Lab EP processing system, Figure 1 and 2, is designed with the following features:

- Fully automated controls - programmable logic controller with a personal computer interface

- Processing horizontally while rotating

- Rinsing horizontally while rotating

- Draining vertically for the electrolyte and rinse water

- Straight aluminium cathode with electrolyte fill ports located at cell centres

- Tooling variable up to a 60 inch cavity length

- Direct current power supply rated at 50 volts and 600 amperes

- Onboard chemical sump that holds 70 gallons of electrolyte and an onboard heat exchanger

- Six ton chiller for electrolyte temperature control

- An integrated $\mathrm{CO}_{2}$ fire suppression system that covers all compartments

The following are programmed steps for a typical cavity process run:

- Lock door and start nitrogen leak test

- Cavity nitrogen purge

- Filter/mix acid

- Rotate cavity

- Fill/overflow cavity

- Sweep voltage

- Start polishing

- $\quad$ Stop polishing

- Tilt to vertical and drain

- Tilt to horizontal and rinse

- $\quad$ Rinse to Resistivity and $\mathrm{pH}$

- Tilt to vertical and drain

- Tilt to horizontal and unlock doors

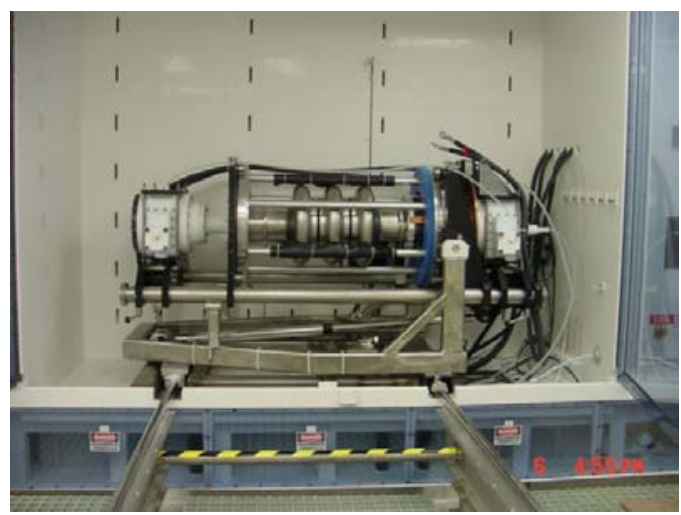

Figure 2. Three Cell $700 \mathrm{MHz}$ Cavity Installed in the EP Tooling.

\section{EARLY TEST RESULTS}

During commissioning of the EP systems, a $700 \mathrm{MHz}$ three-cell cavity was processed to test system functionality and gain understanding of the control system operability. This cavity was processed for $5 \frac{1}{2}$ hours at 350 amperes and 25-40 volts. The material removal as determined by ultrsonic thickness gauging averaged over four samples at each of seven locations along the cavity. The material removal for this cavity was quite uniform with the equators having approximately $20 \%$ less material removed then the beam-tubes. A visual inspection of the cavity showed a smooth interior surface that was bright but not shiny. This cavity was not RF tested.

Two additional cavities were prepared for processing, both high- $\beta$ (HB) prototype cavities built at Jefferson Lab. One of these HB cavities (Figure 5) was modified to allow for small niobium buttons (HB-button) to be inserted at various locations. The second HB prototype cavity (HB-proto) will be used for feedback of RF performance as a function of the process parameters used.

During processing of the HB-button cavity several leaks occurred at the button locations, forcing the run to be ended early. The current was increased up to the 600 amperes and approximately 30 volts during this test and buttons were removed for inspection.

The HB-proto cavity was also processed for four hours at approximately 445 amperes and 18-20 volts. After processing a visual inspection of each end of the cavity interior surfaces showed a difference in appearance from one end to the other. This cavity was then high pressure (HP) rinsed for three hours in the production cleanroom. The cavity was dried over night and all test flanges assembled with the exception of the evacuation flange. 


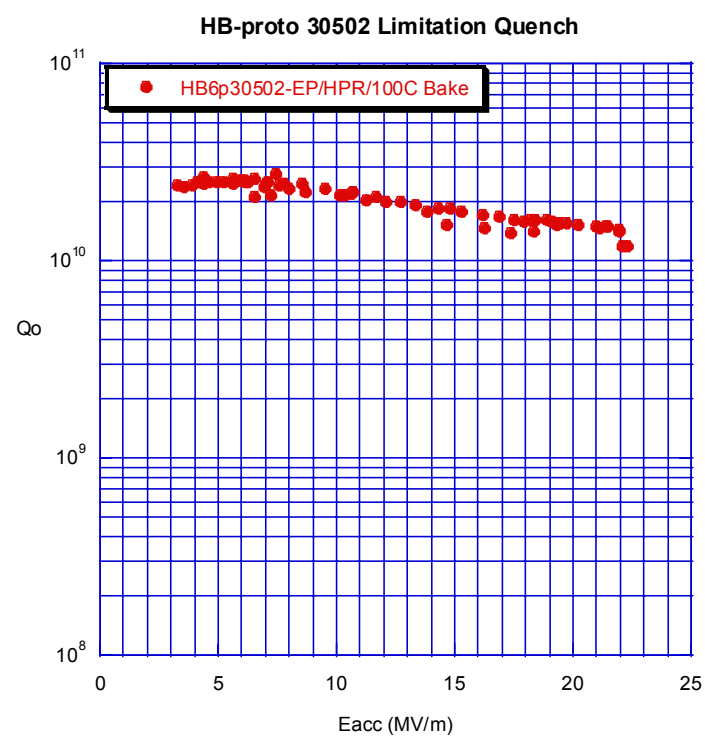

Figure 3. Vertical Test Results of Cavity HBproto.

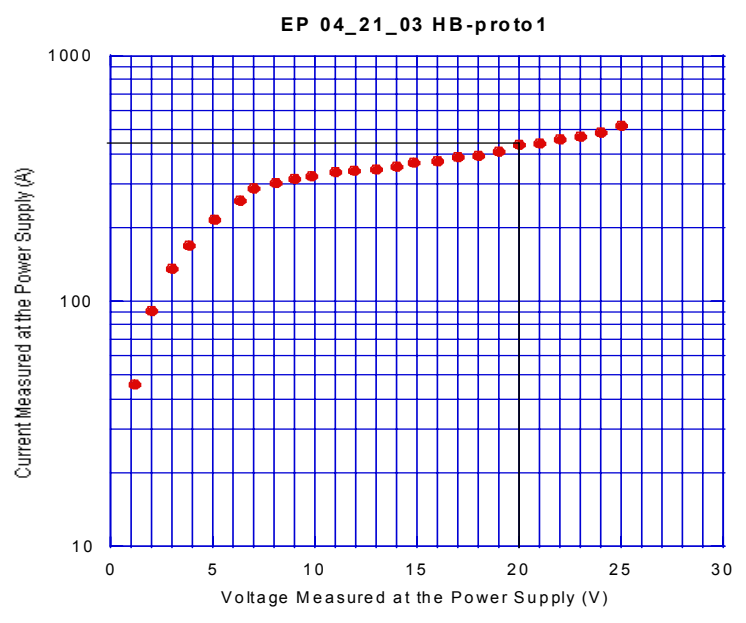

Figure 4. Current-Voltage Curve Taken After Two Hours Into The EP Run.

The cavity was HP rinsed six more hours before assembly of the final flange and evacuation. It was then vacuum baked for 44 hours at $100^{\circ} \mathrm{C}$ and inserted into vertical dewar for RF testing. Figure 3 shows the test result from this cavity with the limitation of a thermal quench at $22.5 \mathrm{MV} / \mathrm{m}$. Figure 4 shows the currentvoltage curve taken after two hours into the run and the operating point for the run. A current-voltage curve was also taken at the beginning of the run before the electrolyte temperature was at $34^{\circ} \mathrm{C}$. This curve did not show the rapidly increasing potential area where process current increases slowly.

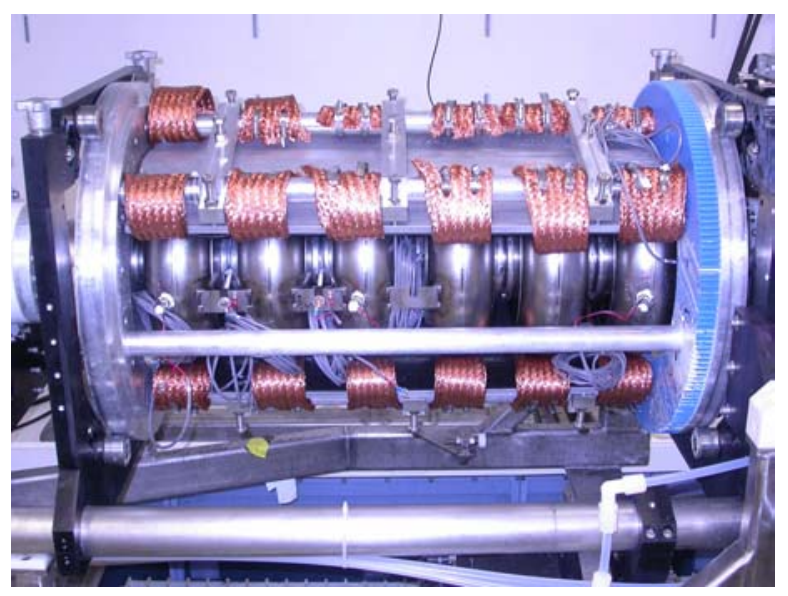

Figure 5. HB-button Cavity Installed Into The Cavity Tilt/Rotation Tooling.

\section{CONCLUSION}

In parallel with production processing of high- $\beta$ cavities, further process optimisation and understanding will be pursued. In particular, surfaces roughness, current density and surface chemistry will be examined at iris, equator, and sidewall locations. The HB-proto cavity (Figure 1) will be alternately processed by EP and BCP and RF tested for comparative evaluation of performance and superconducting parameters. Although existing studies indicate that hydrogen absorption will not be a problem [9], we will verify this through RF testing for the absence of Q-disease.

\section{REFERENCES}

[1] Padamsee, Knobloch, and Hays, "RF Superconductivity for Accelerators", John Wiley and Sons, 1998.

[2] K. Saito, et. al., "Superiority of Electropolishing Over Chemical Polishing on High Gradients", Proceeding of $8^{\text {th }}$ SRF Workshop, Abano, 1997.

[3] E. Kako, et. al., "Improvement of Cavity Performance in the Seclay/Cornell/DESY SC Cavities", $9^{\text {th }}$ SRF Workshop, p.179, SanteFe, 1997.

[4] K. Saito, "High Gradient Performance by Electropolishing with $1300 \mathrm{MHz}$ Single and Multi-cell Superconducting Cavities", ibid p.288..

[5] J. Knobloch, et. al., "High-Field Q Slope in Superconducting Cavities due to Magnetic Field Enhancement at Grain Boundaries", p. 77, 9 ${ }^{\text {th }}$ SRF Workshop. SanteFe, 1999.

[6] N. Steinhau-Kuhl, et. al., "Basic Studies for the Electropolishing Facility at DESY.

[7] Polyflow Engineering Inc., Sylmar, CA

[8] Nomura Plating Co. 1td., Tochigi-ken, Japan

[9] Saito, et. al., "Discovery of the Needless of Outgas Annealing after Horizontally Continuously Rotated Electropolishing with Niobium Superconducting RF Cavities", p. 283, $9^{\text {th }}$ SRF Workshop. SanteFe, 1999. 